\title{
Surfaces de Riemann parfaites en genre 4 et 6
}

\author{
Alexandre Casamayou-Boucau
}

Résumé. Cet article est consacré à la recherche de surfaces de Riemann extrêmes pour la systole, ou tout au moins parfaites. On donne trois nouvelles surfaces de Riemann remarquables en genre 4. Par extension de la méthode, on trouve également une nouvelle surface extrême en genre 6, ainsi qu'une suite infinie de surfaces parfaites non eutactiques de genre $g \geq 4$ quelconque.

Codes AMS (2000). 51M10.

Mots clé. Géométrie hyperbolique, surface de Riemann, systole.

\section{Introduction}

1.1. Position du problème. Soit $X$ une surface de Riemann compacte de genre $g \geq 2$, i.e. une surface hyperbolique orientable fermée. On appelle systole de $X$ la longueur minimale d'une géodésique fermée de $X$ (une telle géodésique n'est jamais homotopiquement triviale). Comme $X$ contient un disque plongé de rayon $\operatorname{Systole}(X) / 2$, on peut aisément majorer la systole en fonction du genre : $\operatorname{Systole}(X)<2 \ln (4 g)$. D'après [Mu], on sait qu'il existe un maximum pour la systole. La détermination de ce maximum est un problème naturel, analogue de la recherche de réseaux euclidiens de densité maximale en géométrie des nombres. Hormis le cas du genre 2, on ne connaît pas le maximum global. Il est donc naturel de rechercher les maxima locaux : une surface réalisant un maximum local sera appelée surface extrême.

Actuellement on ne connaît que peu d'exemples de telles surfaces. En genre 2, la surface de Bolza est la seule surface extrême ([Sc1], [Ba2], [Je]). En genre 3, il y a 3 surfaces extrêmes connues ([Sc1]), dont la quartique de Klein et la courbe de Wiman exceptionnelle. Il est fort probable que ce soient les seules en genre 3, cela reste à montrer toutefois. Ensuite P. Schmutz Schaller a exhibé deux surfaces extrêmes en genre 4, dont une surface triangulaire $M(4)$, et trois surfaces extrêmes en genre 5 ([Sc1], [Sc2]). En genre 6, U. Hamenstädt a récemment trouvé une surface triangulaire extrême $S(13,4)$ ([Ha]). Ensuite viennent quelques autres exemples en genres supérieurs : $S(21,5)$ en genre 10 ([Ha]), $I(x \mid z)$ en genre 11 ([Sc1]), $S_{13}$ en genre 12 ([Sc2]), ainsi qu' une suite infinie $A(n)$ en genre impair supérieur à 7 ([Sc1]). 
Il existe par ailleurs un autre type de surfaces intéressantes à étudier, et plus générales : les surfaces parfaites non extrêmes (pour la définition, cf. §1.2). Les seuls exemples connus sont donnés par P. Schmutz Schaller, qui décrit une famille de telles surfaces pour certains genres supérieurs à 10 ([Sc4]).

Ces considérations s'adaptent au cas non-compact (i.e. les surfaces à bords ou à pointes). Dans certains cas non-compacts, la détermination de la systole maximale a été résolue par P. Schmutz Schaller, par le résultat suivant : les surfaces de Riemann qui correspondent à des sous-groupes principaux de congruences du groupe modulaire sont des surfaces réalisant le maximum global de la systole pour leur signatures respectives (cf. [Sc3]). D'autres exemples sont donnés dans [Ha].

Dans cet article, on s'intéresse exclusivement au cas compact, où la recherche de surfaces extrêmes paraît nettement plus délicate. Le but de ce travail est de trouver de nouveaux exemples en petit genre : on donne une nouvelle surface extrême et deux surfaces parfaites non extrêmes en genre 4 (ce sont les premiers exemples de telles surfaces en petit genre). L'idée de la méthode est de réaliser géométriquement les groupes d'automorphismes à 4 points de ramification, et d'en faire l'étude exhaustive. Par extension, on trouve également une nouvelle surface extrême en genre 6, ainsi

Tableau 1. Surfaces parfaites ou extrêmes connues en genre $g \leq 6$

\begin{tabular}{|c||c|c|c|c|c|}
\hline genre & surface & $($ Systole $) / 2$ & $\sharp\{$ syst $\}$ & propriété & références \\
\hline \hline 2 & $M^{*}$ & 1,52857 & 12 & extrême & (Bolza) [Sc1] \\
\hline \hline 3 & $M(3)$ & 1,99165 & 24 & extrême & (Wiman) [Sc1] \\
\hline 3 & $T(x \mid z)$ & 1,96797 & 21 & extrême & (Klein) [Sc1], rq. 4 \\
\hline 3 & $T(x \mid y)$ & 1,96355 & 22 & extrême & {$[\mathrm{Sc} 1], \mathrm{rq} .4-5$} \\
\hline \hline 4 & $M(4)$ & 2,31225 & 36 & extrême & {$[\mathrm{Sc} 1], \S 3.2$} \\
\hline 4 & $A_{4}$ & 2,30660 & 28 & parfaite & $\S 3.3$ \\
\hline 4 & $C_{4}$ & 2,30159 & 21 & extrême & $\S 2.3$ \\
\hline 4 & $S_{4}$ & 2,26438 & 36 & extrême & {$[\mathrm{Sc} 2], \S 2.2$} \\
\hline 4 & $B_{4}$ & 2,10519 & 24 & parfaite & $\S 4.2$ \\
\hline \hline 5 & $S_{5}$ & 2,45728 & 40 & extrême & {$[\mathrm{Sc} 2], \mathrm{rq.3}$} \\
\hline 5 & $O(x \mid z)$ & 2,44845 & 48 & extrême & {$[\mathrm{Sc} 1], \mathrm{rq.3}$} \\
\hline 5 & $O(x \mid y)$ & 2,37091 & 42 & extrême & {$[\mathrm{Sc} 1], \mathrm{rq} .3$} \\
\hline 5 & $B_{5}$ & 2,16846 & 30 & parfaite & $\S 4.2$ \\
\hline \hline 6 & $I_{6}$ & 2,55450 & 31 & extrême & $\S 5.3$ \\
\hline 6 & $S(13,4)$ & 2,48284 & 39 & extrême & {$[\mathrm{Ha}]$} \\
\hline 6 & $B_{6}$ & 2,20223 & 36 & parfaite & $\S 4.2$ \\
\hline \hline$g \geq 4$ & $B_{g}$ & $<2,27747$ & $6 g$ & parfaite & $\S 4.2$ \\
\hline $2 n-1 \geq 7$ & $A(n)$ & $<2,81698$ & $14 n$ & extrême & {$[\mathrm{Sc} 1]$} \\
\hline
\end{tabular}


qu'une suite infinie de surfaces parfaites non extrêmes de genre $g \geq 4$. En outre, la méthode employée permet de retrouver, de manière unifiée, les surfaces données par P. Schmutz Schaller en genre $g \leq 5$ (cf. remarques 3 à 5). Le tableau 1 récapitule les surfaces remarquables (sous-entendu parfaites ou extrêmes) de genre inférieur à 6 connues à ce jour.

1.2. Surfaces parfaites, surfaces eutactiques. La systole d'une surface de Riemann compacte, marquée, de genre $g$ est définie comme étant le minimum des fonctions longueur géodésique sur l'espace de Teichmüller, fonctions qui sont paramétrées par l'ensemble $C_{g}$ des classes d'homotopie libre de courbes fermées de $\Sigma_{g}$, où l'on exclut les courbes triviales. La fonction systole a récemment été étudiée par P. Schmutz Schaller (cf. [Sc1], [Sc2], [Sc3], [Sc4]) et par Ch. Bavard (cf. [Ba1]).

Soit $T_{g}$ l'espace de Teichmüller des surfaces de Riemann compactes, marquées, de genre $g$; c'est aussi l'espaces des métriques hyperboliques complètes à isotopie près sur une surface orientée $\Sigma_{g}$ de signature $(g, 0)$. On rappelle que $\operatorname{dim}_{\mathbb{R}} T_{g}=6 g-6$. L'espace de Teichmüller sera muni de la métrique de Weil-Petersson. Le groupe modulaire de Teichmüller $\operatorname{Mod}_{g}$ agit sur $T_{g}$ par isométries de Weil-Petersson. Dans la suite, on note $S(X)$ l'ensemble des éléments de $C_{g}$ tels que $l_{c}(X)=\operatorname{Systole}(X)$ ("courbes de longueurs minimales, ou systoles"), où $l_{c}$ désigne la longueur de la géodésique associée à $c$ dans $X$.

Une première caractérisation des surfaces extrêmes a été établie par P. Schmutz Schaller dans [Sc1]. Nous utiliserons ici plutôt la caractérisation donnée par Ch. Bavard dans [Ba1] qui possède l'intérêt de faire apparaitre l'analogie avec la théorie des réseaux.

Nous appellerons surface de Riemann extrême un maximum local de la systole. Nous pouvons également définir, conformément à [Ba1], les notions de surface de Riemann parfaite et de surface de Riemann eutactique de la manière suivante. Soit $X \in$ $T_{g}$; la surface $X$ est dite parfaite si les gradients (pour la métrique de Weil-Petersson) $\left(\nabla_{c}(X)\right)_{c \in S(X)}$ engendrent affinement l'espace tangent $T_{X}\left(T_{g}\right)$; la surface $X$ est dite eutactique si le vecteur nul de $T_{X}\left(T_{g}\right)$ appartient à l'interieur affine de l'enveloppe convexe des gradients $\left(\nabla_{c}(X)\right)_{c \in S(X)}$. On remarque qu'une surface parfaite contient au moins $\operatorname{dim}_{\mathbb{R}} T_{g}+1=6 g-5$ systoles. Ch. Bavard a alors énoncé le résultat suivant, qui est le strict analogue du théorème de Voronoï pour les réseaux :

Une surface de Riemann est extrême si et seulement si elle est parfaite et eutactique ([Ba1]).

On sait que les surfaces de Riemann parfaites (et donc en particulier extrêmes) sont en nombre fini modulo l'action du groupe $\operatorname{Mod}_{g}$, et que leur systole est le logarithme d'un nombre algébrique ([Ba4]). De plus on peut améliorer la borne de la systole par rapport à celle donnée plus haut par l'inégalité : $\cosh (\operatorname{Systole}(X) / 2) \leq$ $\left(2 \sin \frac{\pi}{12 g-6}\right)^{-1}([\mathrm{Ba} 2])$. 
1.3. Méthode mise en œuvre. L'idée qui prélude à la recherche de nouveaux exemples de surfaces extrêmes en petit genre est de considérer comme étant de bons candidats les surfaces qui ont beaucoup d'automorphismes. En effet, si le groupe d'automorphismes est suffisamment gros, on peut espérer obtenir, avec l'action du groupe, un nombre conséquent de systoles, or une surface parfaite contient au moins $6 g-5$ systoles.

Fort de ces considérations, on s'enquiert de la liste des groupes d'automorphismes qui peuvent agir sur une surface de Riemann de genre 4. On trouve une telle classification dans $[\mathrm{Ku}]$ et dans [Bo]. L'article de Bogopol'skiı̌ est cependant le plus riche puisqu'il donne, outre la signature de chaque groupe, l'action de celui-ci sur la surface. Nous avons cherché à étudier de manière quasi-exhaustive les actions des groupes répertoriés dans la table 2 de [Bo, p. 15] : ce sont ceux qui ne sont ni abéliens, ni dihédraux. Pour ce qui est des groupes dihédraux, nous avons examiné ceux d'ordre au moins 8.

Considérons donc un groupe $G$ mentionné dans la table [Bo, pp. 14-15] tel que le quotient de son action soit de genre 0 avec $n$ points de ramification. Plusieurs cas se présentent alors :

- Si $\boldsymbol{n}=\mathbf{3}$, alors il existe une unique surface admettant $G$ comme groupe d'automorphismes : c'est une surface triangulaire. Dans [Sc1], P. Schmutz Schaller indique que parmi toutes les surfaces triangulaires de genre 4, il en existe une seule qui soit extrême : $M(4)$.

- Si $\boldsymbol{n}=\mathbf{4}$, alors il existe une famille de surfaces paramétrée par un unique paramètre complexe, et admettant $G$ comme groupe d'automorphismes. Une fois acquise l'existence d'une telle famille (par des critères purement algébriques), il reste toutefois la délicate question de savoir comment la réaliser géométriquement. Une première idée est de partir d'un graphe connexe trivalent de "genre" 4 (ici, le genre du graphe est entendu comme étant son nombre cyclomatique) : on place alors, à chaque sommet de ce graphe, un pantalon dont les 3 bords ont même longueur ; puis on les recolle suivant le tracé des arêtes du graphe, en effectuant partout le même twist (intuitivement, on épaissit les arêtes). On obtient de la sorte une famille de surfaces invariantes par un certain groupe $G$, paramétrée par le couple de réels $(l, y)=$ (twist,longueur) ou, équivalemment, par un nombre complexe appartenant au demi-plan de Poincaré $(z=l+i y)$. Le groupe $G$ en question a donc une signature comptant 4 points de ramification : il ne reste plus qu'à le retrouver dans la table de [Bo]. En vue d'appliquer cette méthode, on fait donc l'inventaire des graphes trivalents de genre 4.

Cette méthode appliquée au graphe biparti $K_{3,3}$ redonne une famille de surfaces invariantes par $\mathfrak{S}_{3} \times \mathfrak{S}_{3}$ (où $\mathfrak{S}_{3}$ désigne le groupe des permutations d'un ensemble à 3 éléments), famille en partie étudiée par P. Schmutz Schaller à partir d'une construction différente : c'est l'objet du paragraphe 2. Outre la surface extrême $S_{4}$ mentionnée par P. Schmutz Schaller, on met ici en évidence une nouvelle surface extrême $C_{4}$. Par 
ailleurs, on décompose le demi-plan $(l, y)$ en "cellules", où chaque cellule est associée à une classe de géodésiques : si pour une valeur donnée de $(l, y)$ on se trouve dans telle cellule, cela signifie que les géodésiques associées à cette cellule réalisent alors la systole. Les maxima de la systole se trouvent nécessairement parmi les sommets de cette décomposition du demi-plan.

Il reste que l'idée de partir de graphes trivalents est assez limitative, et il n'est pas clair que l'on puisse de la sorte atteindre tous les groupes à 4 points de ramification répertoriés dans $[\mathrm{Bo}]$. On cherche alors à partir de graphes de valence au moins trois, en mettant éventuellement du genre aux sommets.

On peut par exemple considérer le graphe formé par 5 arêtes joignant deux sommets : la surface obtenue est alors le double twisté d'une surface de signature $(0,5)$ ayant la symétrie d'un pentagone régulier. Toutefois, cette famille ne semble pas contenir de surface parfaite $\left([\mathrm{Ca}]^{1}\right)$.

Plus intéressant est le cas du trèfle de genre $4:$ on part d'une surface de signature $(0,8)$ dont on identifie les côtés deux à deux. Si la surface de signature $(0,8)$ a la symétrie du cube, on obtient une famille de surfaces fixées par $\mathfrak{S}_{4}$, le groupe des permutations d'un ensemble à 4 éléments. Elle contient donc forcément la surface de Bring (dont le groupe d'automorphismes est $\mathfrak{S}_{5}$ ) et $M(4)$ (dont le groupe d'automorphismes est $\mathfrak{S}_{4} \times \mathbb{Z}_{3}$ ). Ce fait, déduit de considérations purement algébriques, sera redémontré par une méthode géométrique au §3. De plus on mettra en évidence l'existence, dans cette famille, d'une surface parfaite non eutactique $A_{4}$.

Toujours à partir du graphe du trèfle, si la surface de signature $(0,8)$ a la symétrie de l'octogone régulier, on obtient une famille de surfaces fixées par le groupe dihédral $D_{8}$, d'ordre 16. On n'a pu y déceler de nouvelle surface intéressante ([Ca]).

Pour terminer l'étude exhaustive des actions mentionnées dans la table 2 de [Bo, p. 15], il reste à examiner l'action du groupe de quaternions $Q_{8}$ (mais cette action est moins intéressante puisqu'elle donne lieu à une famille de surfaces hyperelliptiques) ainsi que l'action de $\left(\mathbb{Z}_{3} \times \mathbb{Z}_{3}\right) \triangleleft\langle B\rangle$ (celle ne contenant pas $\left.\mathfrak{S}_{3} \times \mathfrak{S}_{3}\right)$. Pour cette dernière action, nous n'avons pu déceler de surface intéressante ([Ca]).

- Si $\boldsymbol{n}=\mathbf{5}$, on obtient une famille paramétrée par deux nombres complexes. Il faut alors fixer un des deux paramètres en fonction de l'autre pour faire une étude géométrique du même type que celle menée précédemment. A ce stade, on ne peut plus espérer mener une étude exhaustive. Des exemples intéressants se présentent néanmoins, comme celui de la surface associée au graphe fixé par le groupe dihédral $D_{4}$, d'ordre 8 . On opère cette fois-ci un découpage "mixte" de la surface en quatre pantalons et une surface de signature $(0,4)$. L'étude menée au paragraphe 4 révèle l'existence d'une surface parfaite $B_{4}$. En outre cette construction se généralise en genre quelconque et permet de construire une suite infinie de surfaces parfaites et non eutactiques $B_{g}$ de genre $g \geq 4$ quelconque.

\footnotetext{
${ }^{1}$ L'étude des familles mentionnées dans cette introduction, mais dont on n'a pu extraire de nouvelle surface intéressante, est détaillée dans ma thèse.
} 
En dernier paragraphe, on donne un exemple en genre 6. En effet, la construction du $\S 3$, qui consiste à recoller les bords opposés d'une surface de signature $(0,8)$ possédant la symétrie du cube, peut s'adapter au cas de l'icosaèdre : on obtient une famille de genre 6, paramétrée par un unique paramètre complexe, et ayant pour groupe d'automorphismes le groupe alterné de degré 5 (on peut d'ailleurs obtenir une présentation duale de cette famille en épaississant les arêtes du graphe de Petersen). On montre notamment que cette famille contient une surface extrême notée $I_{6}$. A noter qu'on pourrait espérer que cette construction effectuée à partir du dodécaèdre puisse donner une surface intéressante en genre 10, toutefois l'étude de ce cas montre qu'il n'en n'est rien ([Ca]).

\section{Une famille modelée sur $K_{3,3}$}

2.1. Groupe d'automorphismes. On s'intéresse au graphe $K_{3,3}$ (cf. Figure 2.1a) :

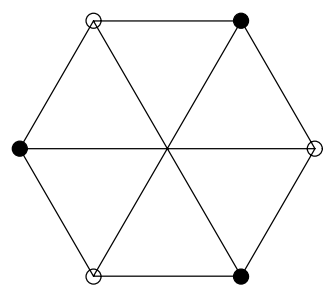

Figure 2.1a

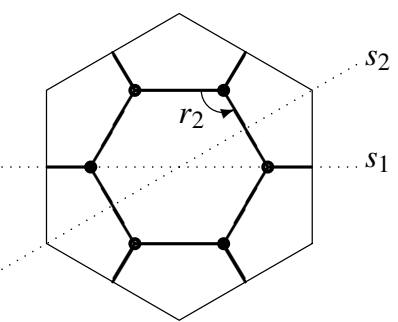

Figure $2.1 b$

Son groupe d'automorphismes $G$ est engendré par deux retournements $s_{1}$ et $s_{2}$ (dont les axes respectifs passent tous deux par le centre du graphe et forment entre eux un angle de $\pi / 6$; le retournement $s_{1}$ passe en outre par un sommet) ; et une rotation $r_{2}$ (de centre l'un des sommets situé sur l'axe de $s_{1}$, d'angle $2 \pi / 3$ ). On note par ailleurs $r_{1}=s_{2} \circ s_{1}$ la rotation de centre le centre du graphe, d'angle $\pi / 3$ (cf. Figure $2.1 \mathrm{~b}$, où le graphe $K_{3,3}$ est dessiné sur le tore). Le groupe $G$ est d'ordre 36 . On remarque qu'il est isomorphe au groupe $\mathfrak{S}_{3} \times \mathfrak{S}_{3}$. En effet,

$$
G=\left\langle s_{1}, s_{2}, r_{2}\right\rangle=\left\langle s_{1}, s_{2}, r_{1}, r_{2}\right\rangle=\left\langle r_{1}^{2}, r_{1} s_{2} r_{1}^{-1}\right\rangle \times\left\langle r_{1}^{2} r_{2}^{2}, r_{1}^{3}\right\rangle \simeq \mathfrak{S}_{3} \times \mathfrak{S}_{3} .
$$

Une fois ce graphe donné, on épaissit les arêtes : on obtient alors une surface de Riemann. Cela revient à placer en chaque sommet du graphe un pantalon dont les trois bords ont même longueur et à les recoller les uns les autres selon un twist identique. Ce procédé nous fournit une famille de surfaces de Riemann paramétrée par deux réels : la longueur du bord et le twist. 
D'après [B-L], on peut décrire cette famille comme intersection d'une quadrique et d'une cubique dans $\mathbb{P}^{3}$ :

$$
C_{(\alpha, \beta)}\left\{\begin{array}{l}
x_{0} x_{1}+x_{2} x_{3}=0, \\
\alpha^{3}\left(x_{0}^{3}-x_{1}^{3}\right)+\beta^{3}\left(x_{2}^{3}-x_{3}^{3}\right)=0,
\end{array}\right.
$$

où le paramètre $(\alpha, \beta)$ décrit $\mathbb{P}^{1}$. On se convainc facilement que $\operatorname{Aut}\left(C_{(\alpha, \beta)}\right) \simeq$ $\mathfrak{S}_{3} \times \mathfrak{S}_{3}$, tant que $(\alpha, \beta) \neq(1, \pm 1)$. Ce groupe noté $G(9 \times 4)$ dans $[\mathrm{Ku}]$, et $\left(\mathbb{Z}_{3} \times \mathbb{Z}_{3}\right) \triangleleft\langle A, B\rangle$ dans [Bo], a pour signature [2, 2, 2, 3]. Dans le cas particulier $(\alpha, \beta)=(1, \pm 1)$, il faut ajouter un automorphisme qui porte l'ordre du groupe d'automorphismes à 72 : c'est un groupe triangulaire $(2,4,6)$, appelé $G(9 \times 8)$ dans $[\mathrm{Ku}]$.

2.2. Une esquisse du graphe de la famille. Cette famille a déjà été étudiée en partie par P. Schmutz Schaller dans [Sc2] à partir d'une présentation différente : il considère en effet le double twisté d'une surface $M_{6}$ de signature $(1,3)$; si bien qu' au lieu de faire les twists selon les bords des pantalons (i.e. les géodésiques $x$ ) comme le suggérait la méthode proposée au premier paragraphe, il exécute les twists selon trois géodésiques $y$ correspondant à des cycles de longueur 6 du graphe. Cela simplifie les calculs puisqu'il y a moins de géodésiques $y$ que de géodésiques $x$ : les effets du twist sont alors plus faciles à calculer. On adoptera donc ce point de vue dans cette étude. La demi-longueur du twist sera notée $l$.

N.B. Dans toute la suite, on notera aussi y la demi-longueur d'une géodésique $y$, et $Y$ la famille des géodésiques $y$. On procédera de même pour toutes les familles de géodésiques fermées définies ci-dessous.

On cherche à déterminer en chaque point du demi-plan $(l / y, y)$ la systole de la surface associée : ceci revient à dessiner un graphe dont les arêtes correspondent à des surfaces dont la systole est réalisée simultanément par deux familles de géodésiques, et dont les sommets correspondent à des surfaces dont la systole est réalisée simultanément par trois familles de géodésiques au moins. On remarque tout d'abord que l'on peut se restreindre au domaine d'étude $l / y \in[0,1 / 2]$ en utilisant certaines symétries du graphe. Il est clair que deux surfaces obtenues à partir d'un twist opposé seront isométriques, ce qui permet de se restreindre au cas $l>0$. Par ailleurs, l'action d'un twist entier selon $Y$ envoie la géodésique $x$ sur une géodésique $x_{1}$ de longueur $2 \arg \cosh [\cosh (t / 2) \cosh (y-l)]$ tout en laissant $y$ invariante. De manière plus générale, un twist de $k \in \mathbb{Z}$ tours selon $Y$ envoie la géodésique $x$ sur la géodésique $x_{k}$, avec $x_{k}(l)=x(l-k y)$. De même pour les géodésiques $u, v$, etc. Le twist entier selon $Y$ translate donc $(l / y, y)$ en $(l / y-1, y)$, et on peut à présent se restreindre au domaine $0 \leq l / y \leq 1 / 2$.

On donne maintenant les valeurs explicites des différentes géodésiques qui sont repésentées sur les figures suivantes et qui nous servirons à construire une partie du 
graphe. Dans ces figures, les deux parties de la surface séparées par $Y$ sont superposées; pour chacune d'elles, les identifications s'effectent par des translations qui échangent les côtés opposés. Les géodésiques fermées sont représentées en trait plein dans la "partie supérieure" et en trait pointillé dans la "partie inférieure". A l'aide des formules de trigonométrie hyperbolique (cf. par exemple [Bu, ch. 2]), on trouve successivement :

$$
\cosh t=\frac{\cosh y / 3}{\cosh y / 3-1},
$$

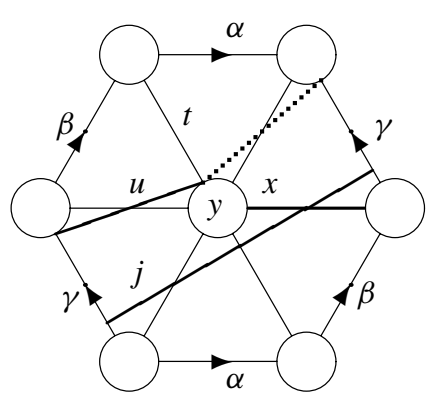

Figure 2.2a

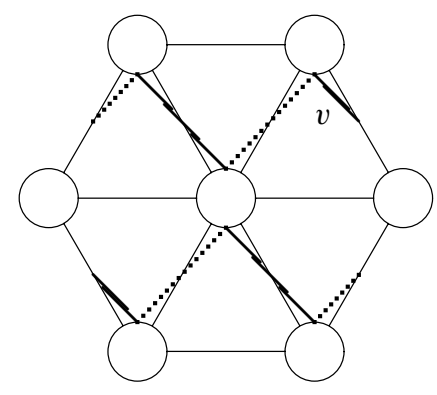

Figure 2.2b

$$
\begin{aligned}
& \cosh j=\left(2 \cosh \frac{y}{3}+1\right) \cdot \cosh \frac{y}{3}, \cosh \frac{x}{2}=\cosh \frac{t}{2} \cdot \cosh l, \\
& \cosh \frac{u}{2}=\cosh \frac{t}{2} \cdot \cosh \left(\frac{y}{3}-l\right), \quad \cosh \frac{v}{6}=\cosh \frac{t}{2} \cdot \cosh \left(\frac{y}{6}-l\right),
\end{aligned}
$$

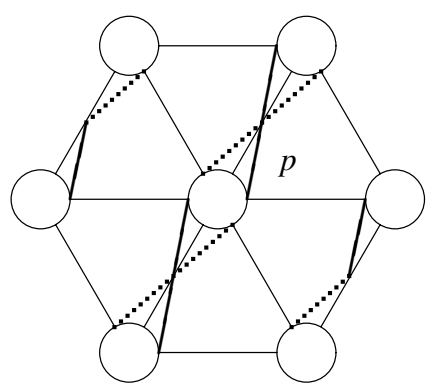

Figure 2.3a

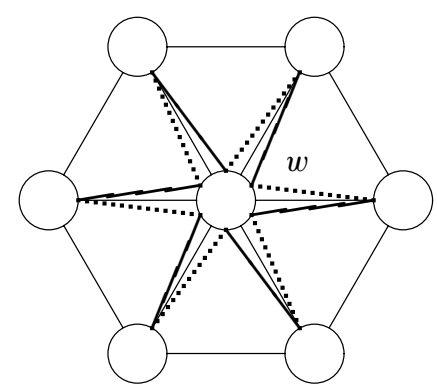

Figure 2.3b

$$
\begin{aligned}
\cosh \frac{p}{6} & =\cosh \frac{t}{2} \cdot \cosh \left(\frac{y}{2}-l\right) \\
\cosh \frac{w}{6} & =\cosh t \cdot \cosh \left(\frac{y}{6}-l\right) \cdot \cosh l-\sinh \left(\frac{y}{6}-l\right) \cdot \sinh l
\end{aligned}
$$




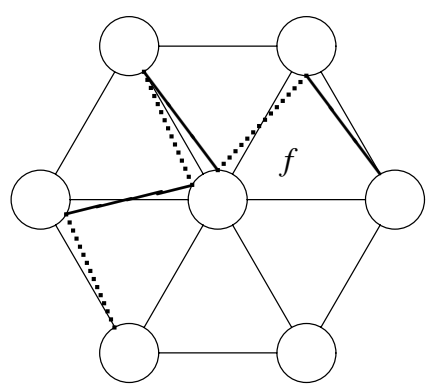

Figure 2.4a

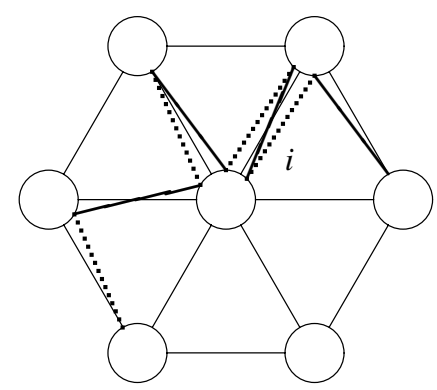

Figure 2.4b

$$
\begin{aligned}
\cosh \frac{f}{2}= & \cosh \frac{t}{2} \cosh \left(\frac{y}{3}-2 l\right) \cosh t \cosh l \\
& -\cosh \frac{t}{2} \sinh \left(\frac{y}{3}-2 l\right) \sinh l+\sinh t \sinh \frac{t}{2} \cosh l
\end{aligned}
$$

On pourrait également exprimer $\cosh i$ (avec une formule plus longue).

Pour chaque famille de géodésiques, on donne ci-dessous le nombre de géodésiques contenues dans cette famille.

\begin{tabular}{|c|c|c|c|}
\hline famille & $Y, V, W, P$ & $J, I$ & $X, U, F$ \\
\hline$\sharp$ & 3 & 18 & 9 \\
\hline
\end{tabular}

Une fois ces calculs acquis, on construit le graphe de la manière suivante : on part de la surface extrême $S_{4}$. On trace alors les trois arêtes issues de ce point, définies par l'égalité de deux familles de systoles choisies parmi les trois familles définissant le point en question (par exemple, si on part de $S_{4}$, on trace $x=u, x=j, u=j$ ). On prolonge ces arêtes jusqu' au moment où une autre géodésique vient réaliser la systole : on arrive alors sur un autre sommet du graphe, d'où partent deux autres arêtes auxquelles on applique de nouveau ce procédé, et ainsi de suite.

La difficulté qui se présente est celle de vérifier qu'à chaque nouveau sommet, la nouvelle géodésique $a$ réalise effectivement la systole. Pour des valeurs de $y$ petites (c'est-à-dire $y \leq 6,5$ ), le rapport $2 a / t$ reste raisonnable (de l'ordre de 6 au plus), donc il est possible d'examiner les longueurs des géodésiques intersectant $Y$ au plus 6 fois, et donc de décider si $a$ est oui ou non la systole. Au delà, $t$ décroît trop vite pour rendre ce type de vérification effective. On donne à présent l'allure qualitative du graphe, pour des valeurs de $y$ n'excédant pas 6 : 


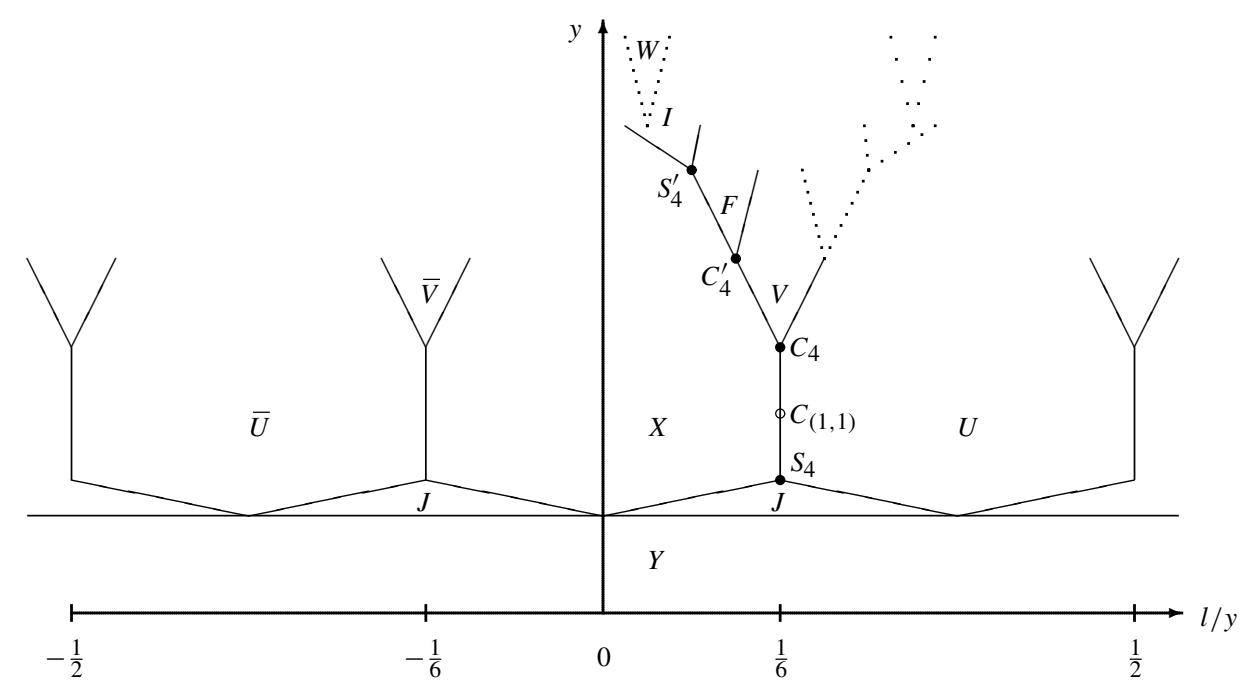

Figure 2.5

Des symétries supplémentaires apparaissent dans le graphe qui s'expliquent ainsi : tout d'abord, on constate en examinant les équations donnant les valeurs de $x, u, v, \ldots$, qu'on a (toujours dans le plan $(l / y, y))$ une symétrie d'axe $l / y=1 / 6$.

Par ailleurs, si on regarde l'effet d'un twist entier selon $X$ sur l'ensemble des géodésiques, on constate qu'on a l'action décrite dans la table suivante (ici, $\bar{u}$ désigne

\begin{tabular}{|c|c|c|c|c|c|c|c|c|}
\hline avant twist & $X$ & $W$ & $Y$ & $U$ & $F$ & $V$ & $I$ & $J$ \\
\hline après twist & $X$ & $Y$ & $\bar{W}$ & $\bar{F}$ & $\bar{U}$ & $\bar{V}$ & $J$ & $\bar{I}$ \\
\hline
\end{tabular}

la géodésique dont la longueur vaut, pour un twist donné $l, \bar{u}(l)=u(-l)$ ). Ainsi donc, si on combine un twist entier selon $X$ avec une inversion du sens du twist effectué selon $Y$ (i.e. une symétrie d'axe $l=0$ sur le graphe), on complète le graphe pour des valeurs de $y>6$.

On remarque une surface intéressante $C_{4}$, caractérisée à isométrie près par $x=$ $u=v$, et dont on montrera l'extrémalité au paragraphe $\$ 2.3$.

D'après les considérations de symétries énumérées plus haut, on retombe sur des surfaces isométriques aux surfaces extrêmes déjà trouvées : une surface $C_{4}^{\prime}$, correspondant au sommet $x=f=v$, qui est isométrique à $C_{4}$; et une surface $S_{4}^{\prime}$, correspondant au sommet $x=f=i$, qui est isométrique à $S_{4}$. Ceci est corroboré par le calcul. Il est donc tout à fait vraisemblable que le graphe ne contienne pas d'autres surfaces extrêmes non isométriques à celles déjà mentionnées; mais ce fait reste à prouver. 
2.3. La surface $\boldsymbol{C}_{\mathbf{4}}$ est extrême. Dans ce paragraphe, on se limite à l'étude de la sous-famille $x=u$, ce qui revient à prendre un twist $2 l$ égal à $y / 3$. Montrons le résultat suivant :

Théorème 1. Il existe un unique double twisté $C_{4}$ de $M_{6}$ (à isométrie près) vérifiant $x=u=v$. La surface $C_{4}$ réalise un maximum local de la systole dans l'espace de Teichmüller de genre 4. Son ensemble de systoles est exactement $X \cup U \cup V$, et contient donc 21 systoles.

Preuve. Montrons que $F=X \cup U \cup V$ est l'ensemble des systoles de $C_{4}$. Après calculs, on obtient : $\cosh y / 3=2+\sqrt{5}, \cosh t=(3+\sqrt{5}) / 4, \cosh x=(9+5 \sqrt{5}) / 4$ $2 x=s+t$, soit en valeurs approchées :

$$
\begin{gathered}
x=u=v \simeq 2,30159, \quad y \simeq 6,36765, \\
j \simeq 4,38498, \quad t \simeq 0,76720, \quad s \simeq 3,83599 .
\end{gathered}
$$

Soit $a$ une systole de $C_{4}$. Alors le nombre $N(a)$ d'intersections de $a$ avec $Y$ vaut au plus $2 v / t=6$. En comparant les valeurs de $y$ et $j$ (systole de $M_{6}$, cf. [Sc2]), on voit que $N(a) \neq 0$. Donc $2 \leq N(a) \leq 6$. De plus, $a$ n'intersecte pas deux perpendiculaires de longueur $t$ issues d'une même géodésique $y$ (l'angle entre $a$ et $Y$ n'est pas trop petit). Si $N(a)=2$, les deux segments de $a$ sont homotopes aux perpendiculaires de longueur $t$ puisque $s>3$; donc $a \in X \cup U$. Si $N(a)=4$, les calculs montrent que $a>3$. Enfin si $N(a)=6$, forcément $a \in V$ puisque $2 v / t=6$ exactement, et qu'aucune autre géodésique coupant $Y$ six fois n'égale $v$. Donc $F$ est bien l'ensemble des systoles de $C_{4}$.

Montrons à présent que $C_{4}$ est eutactique et parfaite. Pour calculer le rang de l'espace engendré par les gradients des systoles, on utilise la matrice formée par le cosinus des angles d'intersections des systoles entre elles. En effet, d'après Wolpert ([Wo]), si $\sigma$ et $\tau$ sont deux systoles on a $\omega\left(l_{\sigma}, l_{\tau}\right)=\Sigma_{p \in \sigma \cap \tau} \cos \theta_{p}$, où $\omega$ est la forme symplectique de Weil-Petersson et $\theta_{p}$ l'angle entre $\sigma$ et $\tau$ au point $p$. En écrivant la matrice intersection des systoles, on obtient une "matrice de Gram symplectique", dont le rang minore celui de l'espace engendré par les gradients des systoles.

Soit $a=\cos \alpha$, où $\alpha$ est l'angle aigu formé par les géodésiques $x$ et $v$ (qui est le même que celui formé par les géodésiques $v$ et $u$ ), soit $c=\cos 2 \alpha=2 a^{2}-1$, $2 \alpha$ étant l'angle formé par les géodésiques $x$ et $u$ quand elles se coupent sur $v$ et soit $d=\cos \theta$, où $\theta$ est l'angle formé par les géodésiques $x$ et $u$ quand elles se coupent sur $y$.

Après calculs, on trouve : $a=\tanh t / 2(\tanh u / 2)^{-1}=1 / \sqrt{5}, c=-3 / 5$ et $d=\cos \left[\pi-2 \arcsin \left(\sinh t / 2(\sinh u / 2)^{-1}\right)\right]=-(2+\sqrt{5}) / 5$. On détermine ensuite 
la matrice $\Omega$ intersection des systoles, rangées ici dans l'ordre $u, x, v$ :

$$
\Omega=\left(\begin{array}{ccc}
0 & -\Omega_{1} & -\Omega_{2} \\
\Omega_{1}^{t} & 0 & \Omega_{2} \\
\Omega_{2}^{t} & -\Omega_{2}^{t} & 0
\end{array}\right),
$$

où

$$
\Omega_{1}=\left(\begin{array}{lllllllll}
d & 0 & c & 0 & 0 & 0 & 0 & d & c \\
c & d & 0 & 0 & 0 & 0 & c & 0 & d \\
0 & c & d & 0 & 0 & 0 & d & c & 0 \\
0 & d & c & d & 0 & c & 0 & 0 & 0 \\
c & 0 & d & c & d & 0 & 0 & 0 & 0 \\
d & c & 0 & 0 & c & d & 0 & 0 & 0 \\
0 & 0 & 0 & 0 & d & c & d & 0 & c \\
0 & 0 & 0 & c & 0 & d & c & d & 0 \\
0 & 0 & 0 & d & c & 0 & 0 & c & d
\end{array}\right) \quad \text { et } \quad \Omega_{2}=\left(\begin{array}{ccc}
a & a & 0 \\
0 & a & a \\
a & 0 & a \\
0 & a & a \\
a & 0 & a \\
a & a & 0 \\
a & 0 & a \\
a & a & 0 \\
0 & a & a
\end{array}\right) \text {. }
$$

Le calcul du rang donne $\operatorname{rang}(\Omega)=18=6 g-6$. En ajoutant alors aux 18 premières colonnes de $\Omega$ les trois dernières pondérees du coefficient $-(c+d) / a>0$, on obtient un vecteur colonne nul. Comme le rang de $\Omega$ est maximal, ceci entraîne précisément que la surface $C_{4}$ est eutactique. Par suite, rang affine et rang vectoriel des gradients coïncident, et $C_{4}$ est parfaite. D'après le théorème démontré par $\mathrm{Ch}$. Bavard dans [Ba1], et rappelé en introduction, (parfaite et eutactique implique extrême), il s'ensuit immédiatement que $C_{4}$ est une surface extrême.

Remarque 1. Dans la sous-famille $x=u$, on remarque que la systole admet un minimum local entre $S_{4}$ et $C_{4}$ : la surface correspondante possède 18 systoles de demilongueur $2 \arg \cosh \frac{3}{2} \simeq 1,92484$; cette surface n'est autre que la surface triangulaire $(2,4,6)$ notée $C_{(1,1)}$ dans [B-L].

Remarque 2. Cette méthode appliquée au graphe cubique redonne la famille de genre 5 étudiée par P. Schmutz Schaller dans le même article ([Sc2]). On n'a pu y déceler de nouvelle surface intéressante; on y retrouve toutefois la famille $O(y)$ décrite différemment par rapport à [Sc1] : en effet, l'analogue de la géodésique $v$ décrite plus haut permet d'identifier la famille $O(y)$ avec l'arête $x=h$, et de mettre en évidence une surface extrême caractérisée à isométrie près par $x=h=v$, et qui n'est autre que $O(x \mid y)$ (cf. [Ca]). 


\section{Le cube}

3.1. Présentation de la famille. On considère une surface de signature $(0,8)$ ayant la symétrie du cube ; autrement dit, on "écorne" les sommets d'un cube. On nomme $y$ les géodésiques de bord. On identifie alors les bords opposés deux à deux (Figure 3.1a), et on obtient une surface de genre 4, ayant pour groupe d'automorphismes $\mathfrak{S}_{4}$. D'après [Bo], la signature de ce groupe est $[2,2,2,4]$; la famille construite est donc paramétrée par un couple de paramètres réels $(l, y)$, où $l$ désigne le demi-twist selon lequel on recolle les géodésiques $y$.

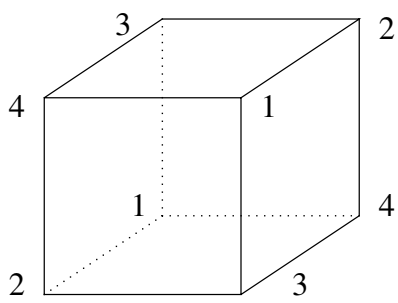

Figure 3.1a

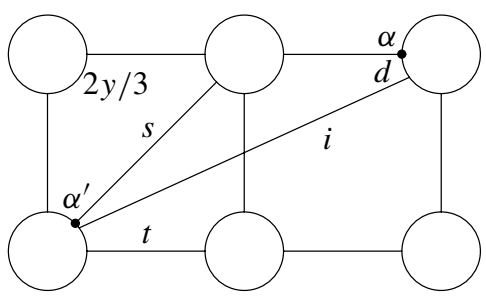

Figure $3.1 \mathrm{~b}$

Toujours d'après la classification de [Bo], on peut conjecturer que cette famille contiendra la surface de Bring (dont le groupe d'automorphismes est $\mathfrak{S}_{5},[\mathrm{R}-\mathrm{R}]$ ), et la surface $M(4)$ (dont le groupe d'automorphismes est $\mathfrak{S}_{4} \times\left\langle x \mid x^{3}=1\right\rangle$, [Sc1]), ce qui est effectivement vérifié dans ce qui suit.

Remarque 3. La même construction effectuée à partir de l'octaèdre (et non plus du cube) redonne la famille $T(y)$ de genre 3 , étudiée dans [Sc1] (cf. [Ca]).

3.2. Une esquisse du graphe. On commence par donner les géodésiques qui interviennent dans la construction du graphe pour $y$ et $l$ pas trop grands.

On note $y$ la géodésique de bord. Soit $t, s$ et $i$ les perpendiculaires représentées sur la figure $3.1 \mathrm{~b}$ (noter que les points $\alpha$ et $\alpha^{\prime}$ sont identifiés quand $l=0$ ). On donne successivement la longueur de ces perpendiculaires, puis pour chaque géodésique représentée ci-après la longueur correspondante :

$$
\begin{gathered}
\sinh \frac{t}{2}=\frac{1}{\sqrt{2} \sinh y / 3}, \quad \sinh \frac{s}{2}=\frac{1}{\tanh y / 3}, \\
\sinh d=\frac{\sinh y \cdot \sinh s}{\sinh i}, \quad \cosh i=\sinh s \cdot \sinh t \cdot \cosh y-\cosh s \cdot \cosh t ;
\end{gathered}
$$



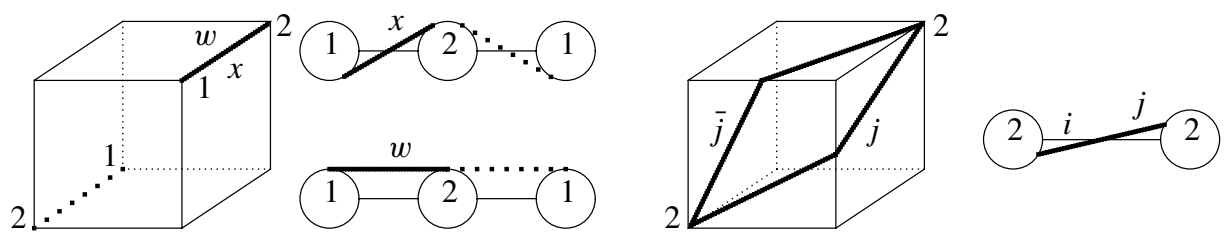

Figure 3.2

$$
\begin{gathered}
\cosh w=\cosh t \cdot \cosh \left(\frac{y}{2}-l\right) \cdot \cosh \left(\frac{y}{2}+l\right)-\sinh \left(\frac{y}{2}-l\right) \cdot \sinh \left(\frac{y}{2}+l\right), \\
\cosh \frac{x}{2}=\cosh \frac{t}{2} \cdot \cosh \left(\frac{y}{2}-l\right), \quad \cosh j=\cosh \frac{i}{2} \cdot \cosh \left(l+\frac{y}{6}-d\right)
\end{gathered}
$$
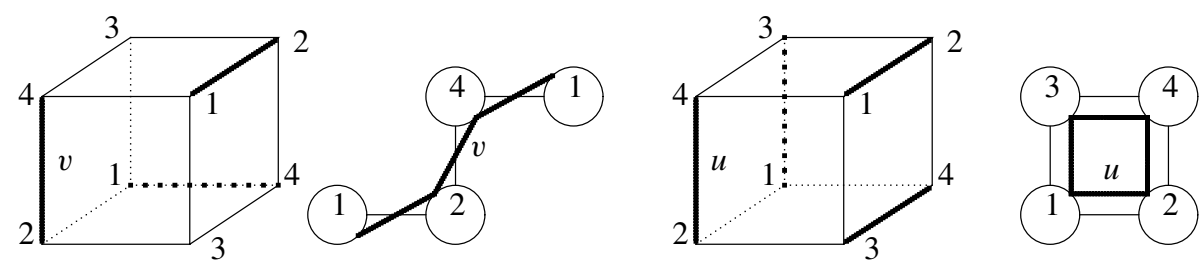

Figure 3.3

$$
\begin{aligned}
\cosh \frac{v}{3} & =\cosh \frac{t}{2} \cdot \cosh \left(\frac{y}{6}-l\right), \\
\cosh \frac{u}{2} & =\cosh t \cdot \cosh \left(\frac{y}{6}-l\right) \cdot \cosh \left(\frac{y}{6}+l\right)-\sinh \left(\frac{y}{6}-l\right) \cdot \sinh \left(\frac{y}{6}+l\right) .
\end{aligned}
$$

On donne dans le tableau ci-dessous le cardinal de chaque famille de géodésiques citées précédemment (ici, $\bar{j}$ désigne la géodésique dont la longueur vaut, pour un twist donné $l, \bar{j}(l)=j(-l))$.

\begin{tabular}{|c|c|c|c|}
\hline famille & $Y, V, \bar{V}$ & $W, J, \bar{J}$ & $U, X, \bar{x}$ \\
\hline$\sharp$ & 4 & 12 & 6 \\
\hline
\end{tabular}

On peut maintenant donner une esquisse du graphe de la famille dans la zone $(y, l / y) \in$ $[0,4] \times[-1 / 2,1 / 2]$. Voici l'allure qualitative du graphe : 


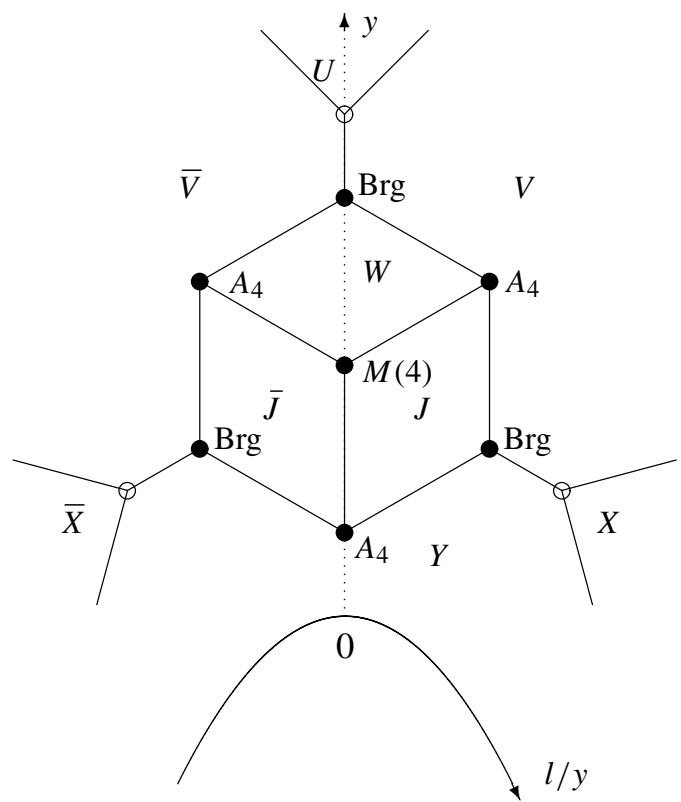

Figure 3.4

Dans cette esquisse, on a "tordu" l'axe des abscisses pour faire apparaître ce qui semble être une symétrie du graphe, car en fait, sur un schéma quantitatif, le cône de $x$ devrait être à peu près axé autour de la droite $l / y=1 / 2$. Néanmoins, on a pris le parti de déformer le graphe puisque, outre la symétrie d'axe $l=0$, il semble apparaître une invariance du graphe par rotation de centre $M(4)$, d'angle $2 \pi / 3$. En effet, le calcul montre que l'on retrouve trois fois, à isométrie près, les surfaces $\operatorname{Brg}:=C_{y=j=v}, A_{4}:=C_{y=j=\bar{j}}$, et $C_{u=v=\bar{v}}$. L'examen du graphe révèle ainsi plusieurs surfaces intéressantes :

- La surface $C_{y=j=v}$ (ou une des deux autres surfaces qui lui sont isométriques : plus précisément $C_{w=v=\bar{v}}$ ou $C_{y=\bar{j}=\bar{v}}$ ): on remarque que cette surface admet exactement 20 systoles, de demi-longueur

$$
y=j=v=3 \arg \cosh (3 / 4+\sqrt{5} / 4) \simeq 2,301592 .
$$

En outre, ses systoles pavent la surface en carrés et pentagones, et se coupent toutes selon le même angle $\alpha=\arccos (2 \sqrt{5} / 11-3 / 11) \simeq 1,436563$. C'est donc la surface de Bring (cf. [R-R]), qui est eutactique et non parfaite. Elle est notée Brg sur le graphe.

- La surface $C_{w=j=\bar{j}}$, qui admet exactement 36 systoles, de demi-longueur

$$
w=j=\bar{j}=\arg \cosh \frac{5+3 \sqrt{3}}{2} \simeq 2,312250 .
$$


On retrouve donc la surface extrême notée $M(4)$ dans [Sc1].

- La surface $C_{w=j=v}$ (ou une des deux autres surfaces qui lui sont isométriques : plus précisément $C_{w=\bar{j}=\bar{v}}$ ou $C_{y=\bar{j}=j}$ ): cette surface admet exactement 28 systoles, de demi-longueur

$$
w=j=v \simeq 2,30659999071 .
$$

Cette surface que l'on notera $A_{4}$ sera étudiée dans le paragraphe $\S 3.3$; on montrera notamment que c'est une surface parfaite non eutactique.

- La surface $C_{y=x=v}$ (ou une des deux autres surfaces qui lui sont isométriques : plus précisément $C_{y=\bar{x}=\bar{v}}$ ou $C_{u=v=\bar{v}}$ ) : cette surface admet exactement 14 systoles, de demi-longueur

$$
y=x=v \simeq 2,29347051505 .
$$

Comme $14<6 g-5=19$, cette surface ne peut être parfaite.

Remarquons par ailleurs que, tout comme dans les sections précédents, on a une invariance du graphe par translation de longueur 1 le long de l'axe des abscisses ; ainsi, cette allure de graphe se transporte au niveau de l'axe $l / y=1$, puis $l / y=2$, etc. De manière plus globale, on obtient donc le graphe suivant (comme précédemment, les géodésiques affectées d'un indice se déduisent des autres par l'effet d'un twist entier selon $Y$; on a par exemple : pour toutes $\left.k \in \mathbb{Z}, j_{k}(l)=j(l-k y)\right)$ :

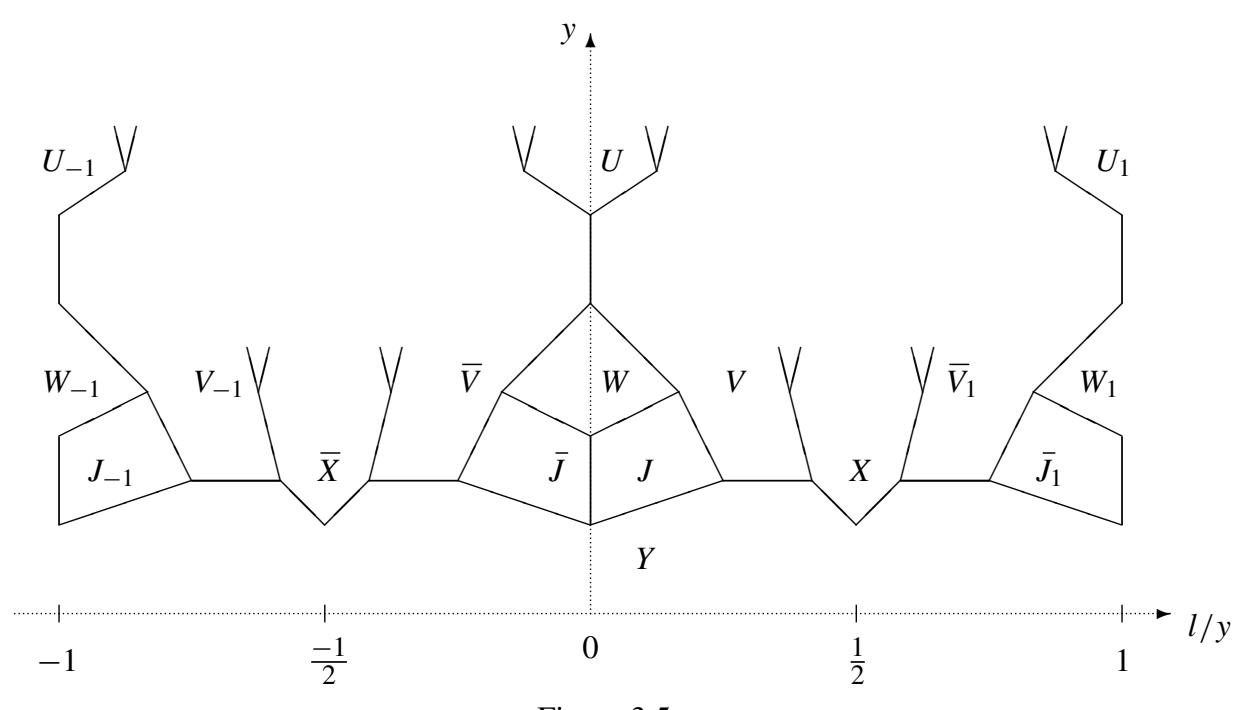

Figure 3.5

3.3. La surface $\boldsymbol{A}_{4}$ est parfaite. Dans ce paragraphe, on s'intéresse à la surface $A_{4}$, définie à isométrie près, par $y=j=\bar{j}$; on montre le résultat suivant :

Théorème 2. $A_{4}$ est une surface parfaite non eutactique. Son ensemble de systoles est exactement $Y \cup J \cup \bar{J}$. 
Preuve. Montrons $Y \cup J \cup \bar{J}$ est l'ensemble des systoles de $A_{4}$. Après calculs, on obtient :

$$
\begin{array}{ll}
y \simeq 2,30659999071, & w \simeq 2,33858918081, \\
u \simeq 3,22774965596, & x \simeq 2,92041491748, \\
v \simeq 2,60164724868, & l=0, \\
t \simeq 1,51944655787, & s \simeq 2,44147924868 .
\end{array}
$$

Soit $a$ une systole de $A_{4}$. Alors le nombre $N(a)$ d'intersections de $a$ avec $Y$ vaut au plus $E(2 y / t)=3$. Donc $0 \leq N(a) \leq 3$. La géodésique $a$ doit appartenir à l'une des familles suivantes $: V \cup \bar{V}(N(a)=3), W \cup X \cup \bar{X}(N(a)=2)$ ou $Y \cup J \cup \bar{J}$ $(N(a) \leq 1)$. Mais $v=\bar{v}>y, w=\bar{w}>y$ et $x>y$. D'où, $a \in Y \cup J \cup \bar{J}$.

Montrons à présent que $A_{4}$ est parfaite. Par un calcul de géométrie hyperbolique, on prouve le lemme suivant :

Lemme 1. Dans un quadrilatère hyperbolique comprenant un angle droit, l'angle $\psi$, opposé à l'angle droit est donné par la formule :

$$
\begin{aligned}
F(a, \alpha ; b, \beta):= & \cos \psi \\
= & -\cos \left(\alpha-\arctan \frac{\tanh a}{\sinh b}\right) \cdot \cos \left(\beta-\arctan \frac{\tanh b}{\sinh a}\right) \\
& +\sin \left(\alpha-\arctan \frac{\tanh a}{\sinh b}\right) \cdot \sin \left(\beta-\arctan \frac{\tanh b}{\sinh a}\right) \cdot \cosh a \cdot \cosh b
\end{aligned}
$$

où a et $b$ désignent les longueurs des deux côtés adjacents à l'angle droit, et $\alpha, \beta$ leurs angles respectivement opposés.

On donne à présent les coefficients qui interviennent dans l'écriture de la matrice intersection des systoles :

$$
\begin{aligned}
& b=\cos \beta=\frac{\tanh (d-y / 6)}{\tanh j} \simeq 0,167373, \\
& e=\cos \epsilon=2 F\left(\frac{t}{2}, \beta ; \frac{y}{2}, \frac{\pi}{2}\right)^{2}-1 \simeq 0,828711, \\
& g=F\left(\frac{t}{2}, \pi-\beta ; \frac{y}{6}, \frac{\pi+\epsilon}{2}\right)=e, \\
& c=F\left(\frac{t}{2}, \beta ; \frac{y}{2}, \frac{\pi-\epsilon}{2}\right) \simeq 0,411716, \\
& m=F\left(\frac{t}{2}, \pi-\beta ; \frac{y}{6}, \frac{\pi-\epsilon}{2}\right) \simeq 0,208497 .
\end{aligned}
$$


En rangeant les sytoles dans l'ordre $\left(y_{1}, \ldots, y_{4}, j_{1}, \bar{j}_{1}, \ldots, j_{12}, \bar{j}_{12}\right)$, la matrice intersection s'écrit :

$$
\Omega=\left(\begin{array}{ccccc}
0 & \Lambda_{1} & \Lambda_{2} & \Lambda_{3} & \Lambda_{4} \\
-{ }^{t} \Lambda_{1} & 0 & \Omega_{1} & \Omega_{2} & -{ }^{t} \Omega_{1} \\
-{ }^{t} \Lambda_{2} & -{ }^{t} \Omega_{1} & 0 & \Omega_{1} & \Omega_{2} \\
-{ }^{t} \Lambda_{3} & \Omega_{2} & -{ }^{t} \Omega_{1} & 0 & \Omega_{1} \\
-{ }^{t} \Lambda_{4} & \Omega_{1} & \Omega_{2} & -{ }^{t} \Omega_{1} & 0
\end{array}\right)
$$

où

$$
\Omega_{1}=\left(\begin{array}{cccccc}
-m & -c & 0 & 0 & 0 & g \\
e & -m & 0 & 0 & 0 & -m \\
-m & g & 0 & 0 & 0 & -c \\
0 & 0 & -g & m & c & 0 \\
0 & 0 & m & -e & m & 0 \\
0 & 0 & c & m & -g & 0
\end{array}\right)
$$

et

$$
\Omega_{2}=\left(\begin{array}{cccccc}
0 & g & -m & -c & 0 & 0 \\
-g & 0 & 0 & 0 & c & m \\
m & 0 & 0 & 0 & m & -e \\
c & 0 & 0 & 0 & -g & m \\
0 & -c & -m & g & 0 & 0 \\
0 & -m & e & -m & 0 & 0
\end{array}\right)
$$

et $\left(\Lambda_{k}\right)_{i, j}=(-1)^{j} \delta_{i}^{k} b(1 \leq i, k \leq 4,1 \leq j \leq 6)$. Le calcul du rang donne $\operatorname{rang}(\Omega)=18=6 g-6$. Par ailleurs, on a une relation entre les colonnes de $\Omega$ : $(2 c-g) \cdot \sum_{1}^{4} C_{y_{i}}+b \cdot \sum_{1}^{12}\left(C_{j_{i}}+C_{\bar{j}_{i}}\right)=0$ avec $4(2 c-g)+24 b \simeq 3,99 \neq 0 ;$ la surface $A_{4}$ est donc parfaite. On a obtenu également l'unique relation (à scalaire près) entre les projections des gradients sur le lieu invariant. D'après [Ba3, Prop. 1.5] (l'eutaxie se lit sur le lieu invariant) on voit que $A_{4}$ n'est pas eutactique puisque $b \simeq 0,17>0$ et $2 c-g \simeq-0,0053<0$.

\section{Une suite infinie de surfaces parfaites}

4.1. Présentation de la famille. Dans ce paragraphe, on montre l'existence d'une surface parfaite non eutactique de genre $g \geq 4$ quelconque.

Soit un entier naturel $g \geq 4$. On s'intéresse à la famille associée au graphe suivant : on considère $g$ sommets formant un polygone régulier à $g$ côtés; les arêtes sont les côtés du polygone; on place ensuite un $(g+1)$-ième sommet au centre du polygone 
que l'on joint aux autres sommets par des arêtes radiales. Dans le cas du genre 4, ce graphe se représente de la manière suivante (Figure 4.1a) :

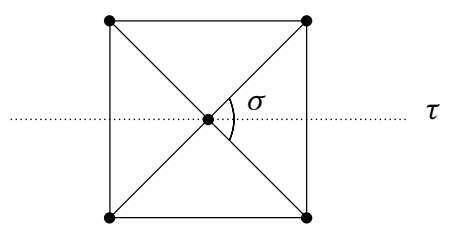

Figure 4.1a

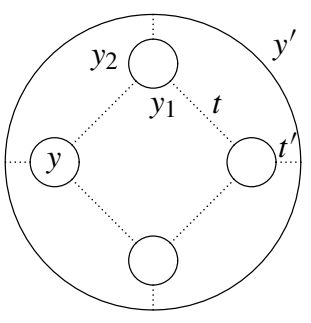

Figure $4.1 b$

Son groupe d'automorphismes $G$ est engendré par un rotation $\sigma$ d'ordre $g$, et un retournement $\tau$ : c'est le groupe dihédral $D_{g}$, d'ordre $2 g$. En genre $g=4$, sa signature est $[2,2,2,2,4]$ d'après [Bo].

La famille obtenue par cette construction est paramétrée par deux paramètres complexes. Pour se ramener à une famille à un paramètre, on va fixer une condition supplémentaire : on découpe la surface suivant les courbes indiquées en pointillés sur la figure $4.1 \mathrm{~b}$, en une surface "centrale" de signature $(0, g)$ et $g$ pantalons. On convient de noter $y$ les $g$ géodésiques indiquées sur la figure $4.1 \mathrm{~b}$, et $y^{\prime}$ la géodésique "extérieure". On note $y_{1}$ la longueur du segment de $y$, contenu dans la sous-surface centrale de signature $(0, g)$, et $y_{2}$ la longueur du segment de $y$, contenu dans un des pantalons. On peut alors paramétrer la famille par les deux couples de réels (twist, longueur) correspondant à la déformation de la surface selon les $g$ géodésiques $y$ et selon la géodésique $y^{\prime}$.

Dans la suite de ce chapitre, on choisit d'imposer $t=t^{\prime}$, ce qui donne alors une famille paramétrée par le seul couple de paramètres réels $(l, y)$, où $l$ est la longueur du demi-twist effectué selon $Y \cup Y^{\prime}$. En effet, $y$ détermine entièrement les longueurs $y_{1}, y_{2}, t=t^{\prime}, y^{\prime}$. Pour obtenir $y_{1}$ et $y_{2}$, il suffit de résoudre le système :

$$
\left\{\begin{array}{l}
y_{1}+2 y_{2}=2 y, \\
\cosh y_{1}-4 \cos ^{2}(\pi / g) \cdot \cosh y_{2}=1-4 \cos ^{2}(\pi / g) .
\end{array}\right.
$$

Ensuite, $t$ et $y^{\prime}$ sont donnés par les formules :

$$
\cosh t=\frac{\cosh y_{2}}{\cosh y_{2}-1} \quad \text { et } \quad 2 y^{\prime}=g y_{2} .
$$

On aurait aussi bien pu se donner $t$ et calculer $y_{1}, y_{2}$ et $y^{\prime}$ en fonction de $t$. 
4.2. Une surface $\boldsymbol{B}_{\boldsymbol{g}}$ parfaite. On note $X$ l'ensemble des $2 g$ géodésiques qui coïncident avec la réunion de deux segments $t$ lorsque le twist est nul (cf. Fig. 4.2). Une telle géodésique a pour demi-longueur : $\cosh \frac{x}{2}=\cosh \frac{t}{2} \cosh l$.

Soit $x \in X$, et soit $y \in Y$ intersectée par $x$, il existe une unique plus petite géodésique simple $z$ telle que $x, y, z$ soient contenues dans une sous-surface de signature $(1,1)$, et telle qu'en outre $z$ intersecte $x$ (resp. $y$ ) une seule fois (cf. Figure 4.2). On note $X^{\prime}$ l'ensemble de ces géodésiques, et $x^{\prime}$ leur demi-longueur, qui vaut : $\cosh x^{\prime}=\cosh t \cosh (y-l) \cosh l-\sinh (y-l) \sinh l$.

Soit $x \in X$ intersectant $y^{\prime}$, il existe une unique plus petite géodésique simple $z$ telle que $x, y^{\prime}, z$ soient contenues dans une sous-surface de signature $(1,1)$, et telle qu'en outre $z$ intersecte $x$ (resp. $y^{\prime}$ ) une seule fois. On note $X^{\prime \prime}$ l'ensemble de ces géodésiques, et $x^{\prime \prime}$ leur demi-longueur : $\cosh x^{\prime \prime}=\cosh t \cosh \left(y^{\prime}-l\right) \cosh l-$ $\sinh \left(y^{\prime}-l\right) \sinh l$.

Pour chaque famille de géodésiques, on donne dans le tableau ci-dessous le nombre de géodésiques contenues dans cette famille.

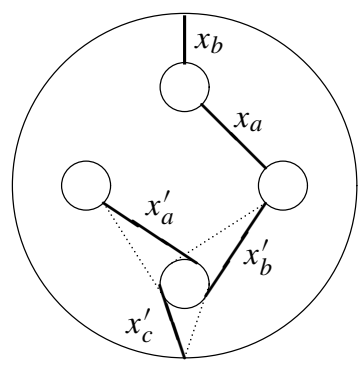

\begin{tabular}{|c|c|}
\hline famille & $\sharp$ \\
\hline$Y, X^{\prime \prime}$ & $g$ \\
\hline$Y^{\prime}$ & 1 \\
\hline$X$ & $2 g$ \\
\hline$X^{\prime}$ & $3 g$ \\
\hline
\end{tabular}

Figure 4.2

Dans la suite, on montre que la surface caractérisée, à isométrie près, par $y=$ $x=x^{\prime}$ est parfaite quelque soit le genre $g>3$.

Remarque 4. En fait la construction menée ici peut se faire dès le genre 3. Cependant ce cas est tout à fait particulier, et c'est pourquoi nous l'avons exclu d'emblée de la discussion. En effet, si $g=3$, on retrouve une surface déjà connue, notée $T(x \mid y)$ par P. Schmutz Schaller (cf. [Sc1]) : le calcul permet d'affirmer que cette surface contient 22 systoles de demi-longueur :

$$
y=y^{\prime}=x=x^{\prime}=x^{\prime \prime} \simeq 1,963546301 .
$$

Ce qui distingue cette surface de celles de genre $g>3$, caractérisées par $y=x=x^{\prime}$, c'est qu'ici l'ensemble des systoles contient en outre $y^{\prime}$ et $x^{\prime \prime}$. Elle est non seulement parfaite, mais encore eutactique (grâce aux systoles additionnelles) donc extrême.

Montrons à présent le résultat suivant : 
Théorème 3. Soit un entier naturel $g>3$. Il existe une unique surface, notée $B_{g}$, caractérisée, à isométrie près, par $y=x=x^{\prime}$. Cette surface est parfaite mais n'est pas eutactique. Son ensemble de systoles est exactement $Y \cup X \cup X^{\prime}$, et contient donc $6 g$ systoles.

Preuve. La condition $y=x=x^{\prime}$ est équivalente au système :

$$
\left\{\begin{array}{l}
\cosh t=\frac{(3 \cosh y+1) \cosh y}{(\cosh y+1)^{2}}, \\
\tanh l=\frac{(-1+\cosh y)}{(1+\cosh y)} \operatorname{coth} y .
\end{array}\right.
$$

On remarque que, à $g$ fixé, la fonction $(3 \cosh y+1) \cosh y /(\cosh y+1)^{2}$ est strictement croissante par rapport à $y$; par ailleurs $y_{1}$ et $y_{2}$ sont des fonctions strictement décroissantes de $t$, donc $y$ est une fonction de $t$ strictement décroissante, donc $t$ est une fonction de $y$ strictement décroissante. Il s'ensuit qu'il existe une unique solution en $y$ à la première équation du système (1).

En outre, la fonction $\operatorname{coth}(y)(-1+\cosh y) /(1+\cosh y)$ est strictement croissante par rapport à $y$, donc $l$ est déterminé de manière unique par la deuxième équation du système (1).

On en déduit qu'il existe un unique couple $(l, y)$ correspondant (pour un genre donné $g$ ) aux conditions $y=x=x^{\prime}$. D'où l'unicité de $B_{g}$.

Montrons que $F=Y \cup X \cup X^{\prime}$ est l'ensemble des systoles de $B_{g}$. On note $s$ la longueur de la perpendiculaire commune (entre deux géodésiques $y$ ), qui soit la plus courte parmi celles qui ne valent pas $t$; on a $: \cosh s=\cosh ^{2} t \cdot \cosh y_{1}-$ $\cosh ^{2} t$. Le tableau suivant donne une estimation des diverses longueurs en fonction du genre. En étudiant les suites $\left(y_{g}\right)_{g>4}$ et $\left(t_{g}\right)_{g>4}$, on remarque qu'elles sont strictement

\begin{tabular}{|c||c|c|c|c|c|}
\hline$g$ & 3 & 4 & 5 & 6 & $+\infty$ \\
\hline \hline$x=x^{\prime}=y$ & 1,963546 & 2,105192 & 2,168463 & 2,202235 & 2,277469 \\
\hline$x^{\prime \prime}$ & 1,963546 & 2,463039 & 3,018964 & 3,601196 & $+\infty$ \\
\hline$y^{\prime}$ & 1,963546 & 2,518569 & 3,099004 & 3,689017 & $+\infty$ \\
\hline$t$ & 1,324921 & 1,376618 & 1,397931 & 1,408875 & 1,432212 \\
\hline$s$ & 1,324921 & 2,335152 & 2,667805 & 2,830168 & 3,379919 \\
\hline$l$ & 0,679361 & 0,743508 & 0,772511 & 0,788072 & 0,822923 \\
\hline $2 y / t$ & 2,96 & 3,06 & 3,10 & 3,13 & 3,18 \\
\hline
\end{tabular}

croissantes et qu'elles tendent vers les valeurs limites portées sur le tableau ci-dessus. En outre, on se convainc assez facilement que $E(2 y / t) \leq 3$. Soit $a$ une systole de $B_{g}$. Alors le nombre $N(a)$ d'intersections de $a$ avec $Y$ vaut au plus $E(2 y / t)=3$. Donc $N(a) \leq 2$. Les valeurs données ci-dessus permettent de conclure. 
On détermine ensuite la matrice $\Omega$ d'intersection des systoles prises dans l'ordre $Y, X_{a}, X_{b}, X_{c}^{\prime}, X_{a}^{\prime}, X_{b}^{\prime}$ (cf. Figure 4.2). On pose

$$
\begin{aligned}
& a=\cos \alpha=\cosh y(\cosh y+1)^{-1}, \\
& c=\cos 2 \alpha=2 a^{2}-1, \\
& e=\sin ^{2} \alpha \cdot \cosh \left(y-y_{1}\right)-\cos ^{2} \alpha, \\
& f=\sin ^{2} \alpha \cdot \cosh \left(y-y_{2}\right)-\cos ^{2} \alpha, \\
& h=\sin \alpha \cdot \sin 2 \alpha \cdot \cosh \left(y-y_{1}\right)-\cos \alpha \cdot \cos 2 \alpha, \\
& k=\sin \alpha \cdot \sin 2 \alpha \cdot \cosh \left(y-y_{2}\right)-\cos \alpha \cdot \cos 2 \alpha .
\end{aligned}
$$

Soit $C\left(\omega_{1}, \ldots, \omega_{g}\right)$ la matrice circulante définie par $C_{i, j}=\omega_{j-i+1}$ (indice modulo $g)$. On a alors $\Omega=\left(\Omega_{i, j}\right)_{1 \leq i, j, \leq 6}$ où les $\Omega_{i, j}$ sont des blocs carrés d'ordre $g$ vérifiant $\Omega_{i, j}+{ }^{t} \Omega_{j, i}=0$ et donnés par :

$$
\begin{array}{llrl}
\Omega_{i, i} & =0 \quad(1 \leq i \leq 4), & \Omega_{5,5} & =-\Omega_{6,6}=C(0,-h, 0, \ldots, 0, h), \\
\Omega_{1,2} & =C(a, 0, \ldots, 0, a), & & \Omega_{1,3}=\Omega_{3,4}=-\Omega_{1,4}=C(a, 0, \ldots, 0), \\
\Omega_{1,5} & =C(c, 0, \ldots, 0,-a), & \Omega_{1,6} & =C(-a, 0, \ldots, 0, c), \\
\Omega_{2,3} & =\Omega_{5,6}=0, & & \Omega_{2,4}=C(f, f, 0, \ldots, 0), \\
\Omega_{2,5} & =C(a, 0, \ldots, 0, e), & \Omega_{2,6} & =C(a, e, 0, \ldots, 0), \\
\Omega_{3,5} & =C(0, \ldots, 0, f), & & \Omega_{3,6}=C(f, 0, \ldots, 0), \\
\Omega_{4,5} & =C(-k, 0, \ldots, 0), & & \Omega_{4,6}=C(0,0, \ldots,-k) .
\end{array}
$$

On montre maintenant que $\operatorname{rang}(\Omega)=6 g-6$ en distinguant deux cas suivant la parité de $g$.

Si $g$ est impair, on pose $M_{4}=\left(\Omega_{i, j}\right)_{1 \leq i, j, \leq 4}$, et $\Omega=\left(\begin{array}{cc}M_{4} & M_{4,2} \\ M_{2,4} & M_{2}\end{array}\right)$. On voit facilement que $M_{4}$ est inversible (par exemple det $M_{4}=2 a^{g}(a-f)^{g} \neq 0$ ); le noyau de $\Omega$ a donc la même dimension que celui de $U=M_{2}+{ }^{t} M_{4,2} M_{4}^{-1} M_{4,2}$. Le calcul montre que $U=\left(\begin{array}{cc}P & 0 \\ 0 & -P\end{array}\right)$ avec $P=C(0, A, B,-B, \ldots, B,-B,-A)$, où

$$
\left\{\begin{array}{l}
A=\frac{1}{a}\left(a^{2}+a c-h a-k a-e a+c f\right), \\
B=\frac{1}{f-a}\left(a c-c e+a^{2}-e a-a f-k a+e f+e k\right) .
\end{array}\right.
$$

Il est élémentaire de vérifier que la matrice antisymétrique $P$ est de rang au moins $g-3$ sauf si $A=B=0$, ce qui n'est pas le cas ici. On conclut que $\operatorname{rang}(\Omega)=6 g-6$. Remarquer que $P$ doit être de rang exactement $g-3$, ce qui traduit des relations entre les réels $a, c, e \ldots$ (par exemple $A^{2}-A B-B^{2}=0$ si $\left.g=5\right)$.

Quand $g$ est pair, on décompose $\mathbb{R}^{6 g}$ suivant certains sous-espaces invariants par l'action de $\Omega$. Soit $u, v \in \mathbb{R}^{g}$ donnés par $u^{j}=1, v^{j}=(-1)^{j+1}(j=1, \ldots, g)$. On 
identifie $\mathbb{R}^{6 g}$ avec $\left(\mathbb{R}^{g}\right)^{6}$ et, pour $x \in \mathbb{R}^{g}$ on note $x_{i}$ le vecteur $(0, \ldots, x, \ldots, 0)(x$ en place $i$ ). On considère la décomposition orthogonale $\Omega$-invariante :

$$
\mathbb{R}^{6 g}=E \oplus F
$$

où $E$ est engendré par les $u_{i}$ et les $v_{i}(i=1, \ldots, 6)$. Le sous-espace $E$ est somme de 4 sous-espaces invariants et on vérifie immédiatement que $\operatorname{rang}\left(\Omega_{\mid E}\right)=10$. Ensuite on note $w^{j} \in \mathbb{R}^{g}$ le $j$-ème vecteur colonne de la matrice ${ }^{t} C(1,-1,1,-1,0, \ldots, 0)$ et on prend $\left(w^{j}\right)_{i} i=1, \ldots, 6, j=1, \ldots, g-2$ comme base de $F$. La matrice $M$ de l'action de $\Omega$ dans cette base est formée de blocs carrés $M_{i, j}$ d'ordre $g-2$ $(1 \leq i, j \leq 6)$. Soit $I=[2,3,5,6]$ et $J=[1,4]$; on note $N_{4}, N_{4,2}, N_{2,4}$ et $N_{2}$ les matrices extraites respectivement de $M$ suivant $I \times I, I \times J, J \times I$ et $J \times J$ (indices des blocs), puis on procède comme dans le cas où $g$ est impair : $N_{4}$ étant inversible, le rang de $M$ se déduit de celui de $V=N_{2}-N_{2,4} N_{4}^{-1} N_{4,2}$. Le calcul montre que $V=\left(\begin{array}{cc}0 & Q \\ -Q & 0\end{array}\right)$, où $Q=\left(q_{i, j}\right)_{1 \leq i, j \leq g-2}$ avec $q_{i, i}=C(1 \leq i \leq g-2), q_{i+1, i}=$ $q_{i-1, i}=D$ pour $2 \leq i \leq g-3, q_{2 r, 1}=q_{2 r-3, g-2}=-D$ pour $2 \leq r \leq g / 2-1$ (les autres $q_{i, i}$ étant nuls) et enfin :

$$
\left\{\begin{aligned}
C= & \frac{1}{f(a-e)}\left(a^{3}+a^{2}[c-f-e-k-2 h]\right. \\
& \left.\quad+a[f e-k e+2 f h+c e+2 f k]-2 c f^{2}\right), \\
D= & \frac{1}{f(a-e)}\left(a f h+a f k-a^{2} h+-k a e-c f^{2}+e a c\right) .
\end{aligned}\right.
$$

Comme $D \neq 0$, le rang de $Q$ vaut au moins $g-4$, donc $\operatorname{rang}(\Omega)=6 g-6$. En fait on a nécessairement $\operatorname{rang}(Q)=g-4$, d'où des relations entre $a, c, e \ldots$ (par exemple $C=0$ si $g=4$ ou $C+D=0$ si $g=6$ ).

Sachant que le rang des gradients est maximal, on peut conclure par un argument d'eutaxie relative (comme au §3) que $B_{g}$ est parfaite et non eutactique. En effet, en affectant d'un même coefficient $\lambda_{i}$ toutes les colonnes correspondantes aux géodésiques d'une même orbite $(i=1, \ldots, 5)$, on trouve une combinaison linéaire nulle (avec $\sum_{i=1}^{5} \lambda_{i} \neq 0$ ) en prenant

$$
\left\{\begin{array}{l}
\lambda_{1}=a^{2}+a e-2 f^{2}>0 \\
\lambda_{2}=a(k-f+a-c)>0 \\
\lambda_{3}=a^{2}+a e+2 f c-2 f a-2 a k<0 \\
\lambda_{4}=a(a+e-2 f)>0 \\
\lambda_{5}=a(a-f)>0
\end{array}\right.
$$

\section{L'icosaèdre}

5.1. Présentation de la famille. On considère une surface de signature $(0,12)$ ayant la symétrie de l'icosaèdre. On nomme y les géodésiques de bords. En identifiant alors 
les bords opposés deux à deux, on obtient une surface de genre 6 , ayant pour groupe d'automorphismes $\mathfrak{A}_{5}$, le groupe alterné de degré 5 (d'ordre 60). En faisant varier la longueur du bord $y$ et le twist $2 l$ selon lequel on recolle les bords opposés, on obtient une famille de surfaces de Riemann, paramétrée par un couple de paramètres réels $(l, y)$. Dans un premier temps, on donne une allure du graphe, puis dans le dernier paragraphe, on montre que cette famille contient une surface extrême.

5.2. Une esquisse du graphe. Dans ce paragraphe, on recense en premier lieu des géodésiques qui coupent l'ensemble des bords au plus trois fois (cette liste sera utilisée pour la construction du graphe comme pour la démonstration du théorème 5), puis on donne les longueurs de deux classes de géodésiques intersectant l'ensemble des bords respectivement 5 et 10 fois : celles-ci nous permettront de compléter le graphe.

On commence par donner les longueurs des perpendiculaires représentées sur la figure Figure 5.1a (les hexagones figurés correspondent aux faces triangulaires de l'icosaèdre) :

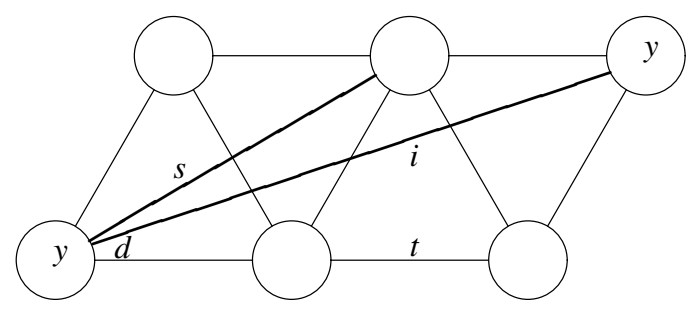

Figure 5.1a

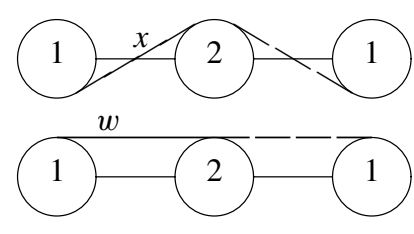

Figure 5.1b

$$
\begin{aligned}
\sinh \frac{t}{2} & =\frac{1}{2 \cdot \sinh (y / 5)}, \quad \cosh \frac{s}{2}=\sinh t \cdot \sinh \frac{2 y}{5}, \\
\sinh d & =\frac{\sinh y \cdot \sinh s}{\sinh i}, \quad \cosh i=\sinh t \cdot \sinh s \cdot \cosh y-\cosh t \cdot \cosh s .
\end{aligned}
$$

Ensuite, on calcule les longueurs de certaines géodésiques qui interviennent dans l'étude de la famille. Pour une géodésique $a$, on note $N(a)$ le nombre d'intersections entre $a$ et l'ensemble des bords $Y$. En outre les bords sont numérotés de la manière suivante : on choisit un sommet que l'on numérote 1 ; ce sommet est entouré de 5 faces triangulaires formant un pentagone; on numérote alors les cinq sommets de ce pentagone de 2 à 6 dans le sens trigonométrique.

Soit $J$ l'esnsemble des géodésiques homotopes au segment $i$. Si $j \in J$, on a $N(j)=1$ et :

$$
\cosh j=\cosh \frac{i}{2} \cdot \cosh \left(d-\frac{y}{10}-l\right) \text {. }
$$

Soit $X_{t}, X_{s}, W_{t}$ et $W_{s}$ les familles de géodésiques définies par la figure $5.1 \mathrm{~b}$ (avec $N(a)=2$ ), l'indice $s$ ou $t$ étant relatif à la perpendiculaire commune entre les deux 
bords. En réalité, la géodésique affectée d'un indice $s$ sera toujours plus grande que la géodésique correspondante, affectée de l'indice $t$. On se bornera donc à donner seulement les valeurs de $x_{t}, w_{t}$, omettant dès lors les indices $t$ :

$$
\begin{aligned}
\cosh \frac{x}{2} & =\cosh \frac{t}{2} \cdot \cosh \left(\frac{y}{2}-l\right), \\
\cosh w & =\cosh t \cdot \cosh \left(\frac{y}{2}-l\right) \cdot \cosh \left(\frac{y}{2}+l\right)-\sinh \left(\frac{y}{2}-l\right) \cdot \sinh \left(\frac{y}{2}+l\right) .
\end{aligned}
$$

On considère également des familles $V_{k}(k=0 . .3)$ de géodésiques avec $N\left(v_{k}\right)=3$ et 3 segments homotopes aux perpendiculaires communes entre les bords (cf. le cas du cube \$3). L'indice que porte $v$ désigne le nombre de segments homotopes à $s$; donc $v_{k}$ possède $(3-k)$ segments homotopes à $t$. Par exemple, la géodésique joignant les bords 1-4-6-1 est dans $V_{0}$. De fait, le calcul montre que $v_{0}$ est toujours la plus petite parmi les quatre types de géodésiques $V_{k}$. Pour exemple, on donnera l'expression de $v_{1}$ (outre celle de $v_{0}$ ). Dans la suite $v$ désigne $v_{0}$.

$$
\begin{aligned}
\cosh \frac{v}{3}= & \cosh \frac{t}{2} \cdot \cosh \left(\frac{y}{10}-l\right), \\
\cosh v_{1}= & \cosh \frac{s}{2} \cosh \left(\frac{2 y}{5}-2 l\right) \cosh t \cosh \left(\frac{3 y}{10}-l\right) \\
& +\cosh \frac{s}{2} \sinh \left(\frac{2 y}{5}-2 l\right) \sinh \left(\frac{3 y}{10}-l\right) \\
& +\cosh \left(\frac{3 y}{10}-l\right) \sinh \frac{s}{2} \sinh t .
\end{aligned}
$$

Les géodésiques suivantes sont définies comme dans le cas du cube (\$3) : la géodésique $z$ possédant 5 segments homotopes à $t$ (par exemple, la géodésique joignant les bords 5-3-6-4-2-5 est de ce type-ci), la géodésique $u$ possédant 10 segments homotopes à $t$ (par exemple, la géodésique joignant les bords 1-6-4-5-3-1-6-4-5-3-1 est de ce type-là). On a $N(z)=5, N(u)=10$ et en outre :

$$
\begin{aligned}
\cosh \frac{z}{5} & =\cosh \frac{t}{2} \cdot \cosh \left(\frac{3 y}{10}-l\right), \\
\cosh \frac{u}{5} & =\cosh t \cdot \cosh \left(\frac{y}{10}+l\right) \cdot \cosh \left(\frac{y}{10}-l\right)-\sinh \left(\frac{y}{10}+l\right) \cdot \sinh \left(\frac{y}{10}-l\right) .
\end{aligned}
$$

Pour chaque famille de géodésiques, on donne ci-dessous le nombre de géodésiques contenues dans cette famille.

\begin{tabular}{|c|c|c|c|c|c|}
\hline famille & $Y, U, Z$ & $V$ & $X$ & $W$ & $J$ \\
\hline$\sharp$ & 6 & 10 & 15 & 30 & 60 \\
\hline
\end{tabular}


Fort de ces calculs, on peut à présent esquisser le graphe de la famille pour $l / y \in$ $[0,1 / 2]$, puis, par les arguments de symétrie habituels, l'étendre horizontalement :

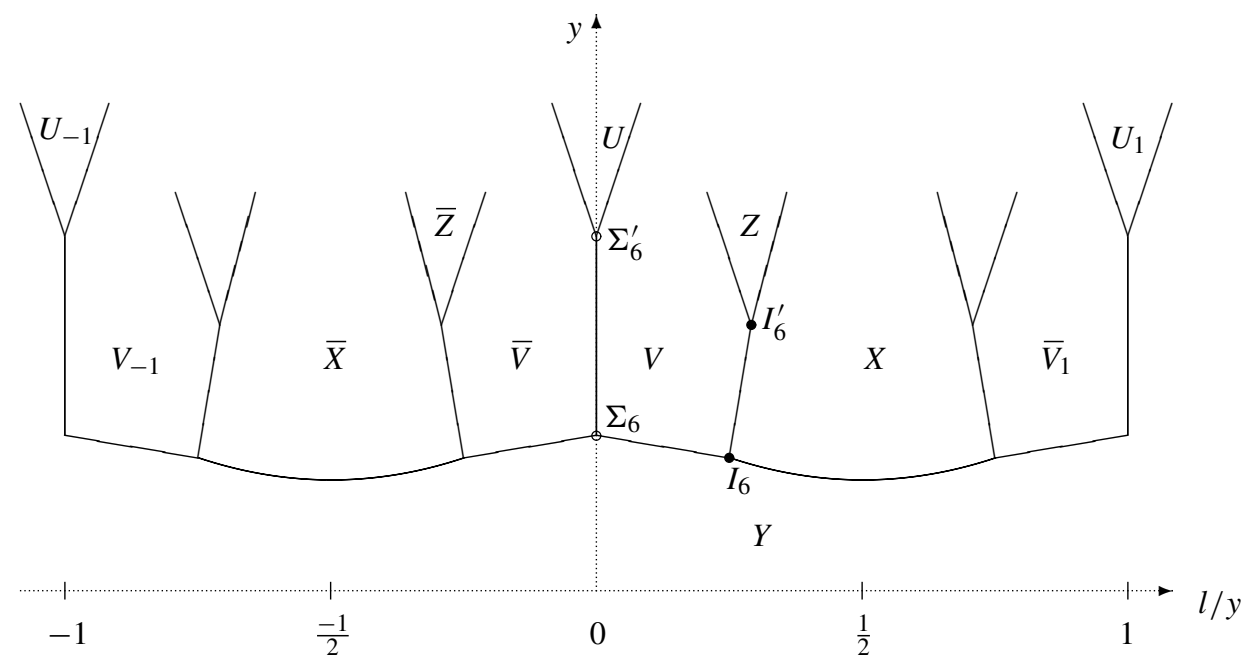

Figure 5.2

Parmi les sommets de ce graphe, on remarque deux surfaces non isométriques :

- La surface $\Sigma_{6}$ définie par $y=v=\bar{v}$ (qui est en outre isométrique à la surface $\Sigma_{6}^{\prime}$ définie par $u=v=\bar{v}$ ) : cette surface admet exactement 26 systoles, de demilongueur

$$
y=v=\bar{v} \simeq 2,608979056 .
$$

Cette surface ne peut pas être parfaite, puisqu'elle possède seulement 26 systoles et que $26<6 g-5=31$.

- La surface $I_{6}$ définie par $y=v=x$ (qui est en outre isométrique à la surface $I_{6}^{\prime}$ définie $\operatorname{par} z=v=x$ ): cette surface admet exactement 31 systoles, de demi-longueur

$$
y=v=x \simeq 2,554500933 .
$$

5.3. La surface $I_{6}$ est extrême. Dans ce paragraphe, on s'attache à montrer le résultat suivant :

Théorème 4. La surface $I_{6}$ caractérisée, à isométrie près, par $y=x=v$, réalise un maximum local de la systole dans l'espace de Teichmüller de genre 6. Son ensemble de systoles est exactement $Y \cup X \cup V$, et contient donc 31 systoles. 
Preuve. Montrons que $F=Y \cup X \cup V$ est l'ensemble des systoles de $I_{6}$. Après calculs, on obtient :

$$
\begin{aligned}
y=x & =v \simeq 2,554500933, \quad w \simeq 2,793330751, \quad j \simeq 3,135994174, \\
t & \simeq 1,672747025, \quad s \simeq 3,599590847, \quad l \simeq 0,3399602340 .
\end{aligned}
$$

Soit $a$ une systole de $I_{6}$. On voit facilement que $a$ doit couper $Y$. Alors le nombre $N(a)$ d'intersections de $a$ avec $Y$ vaut au plus $E(2 y / t)=3$ (puisque en outre $t<(t+s) / 2<s)$. De plus $a$ doit être comprise dans une des familles inventoriées dans le paragraphe précédent. En comparant les valeurs approchées des longueurs de ces différentes géodésiques, on conclut que $a \in Y \cup X \cup V$.

Montrons à présent que $I_{6}$ est eutactique et parfaite. Soit $\alpha$ l'angle aigu formé par les géodésiques $x$ et $y$, soit $\beta$ l'angle aigu formé par les géodésiques $v$ et $y$ et soit $\gamma$ (resp. $\delta$ ) l'angle formé par les géodésiques $v$ et $x$ quand elles se coupent sur $t$ (resp. sur $y$ ). Posons à présent :

$$
\begin{aligned}
& a=\cos \alpha=\frac{\tanh (y / 2-l)}{\tanh x / 2} \simeq 0,823907, \\
& b=\cos \beta=\frac{\tanh (l-y / 10)}{\tanh v / 3} \simeq 0,207432, \\
& c=\cos \gamma=\frac{\cosh x / 2 \cdot \cosh v / 3-\cosh 2 y / 5}{\sinh x / 2 \cdot \sinh v / 3} \simeq 0,698984, \\
& d=\cos \delta=\cos (\pi-\alpha-\beta) \simeq 0,383493 .
\end{aligned}
$$

On détermine ensuite (en fonction de $a, b, c, d$ ) la matrice $\Omega$ d'intersection des systoles entre elles, rangées dans l'ordre $(y, x, v)$. Le calcul du rang donne $\operatorname{rang}(\Omega)=30=$ $6 g-6$. De plus, on a une relation à coefficients tous $>0$ entre les colonnes $C_{j}$ de $\Omega$ : $(c+d) \sum_{j=1}^{6} C_{j}+b \sum_{j=7}^{21} C_{j}+a \sum_{j=22}^{31} C_{j}=0$. Il s'ensuit que $I_{6}$ est une surface extrême.

Remerciements. Je voudrais remercier vivement le Professeur Christophe Bavard pour la qualité des dialogues que nous avons eus au cours de l'élaboration de cet article, ainsi que pour les judicieuses remarques dont il m'a fait part.

\section{Références}

[Ba1] Ch. Bavard, Systole et invariant d'Hermite. J. Reine Angew. Math. 482 (1997), 93-120. Zbl 1011.53035 MR 1427658

[Ba2] Ch. Bavard, La systole des surfaces hyperelliptiques. Prépubl. Ec. Norm. Sup. Lyon 71 (1992). 
[Ba3] Ch. Bavard, Familles hyperboliques de réseaux symplectiques. Math. Ann. 320 (2001), 799-833. Zbl 1008.11027 MR 1857139

[Ba4] Ch. Bavard, Propriétés de finitude pour les familles de réseaux et analogues. Prépubl. Lab. Math. Pures Bordeaux 129 (2001).

[B-L] Ch. Birkenhake, H. Lange, A family of abelian surfaces and curves of genus four. Manuscripta Math. 85 (1994), 393-407. Zbl 0821.14027 MR 1305750

[Bo] O. V. Bogopol'skil̆, Classifying the actions of finite groups on orientable surfaces of genus 4. Siberian Adv. Math. 7 (4) (1997), 9-38. Zbl 0926.57011 MR 1604157

[Bu] P. Buser, Geometry and spectra of compact Riemann surfaces. Progr. Math. 106, Birkhäuser, 1992. Zbl 0770.53001 MR 1183224

[B-S] P. Buser, P. Sarnak, On the period matrix of a Riemann surface of large genus (with an Appendix by J. H. Conway and N. J. A. Sloane). Invent. Math. 117 (1994), 27-56. Zbl 0814.14033 MR 1269424

[Ca] A. Casamayou-Boucau, Surfaces de Riemann parfaites en petit genre. Thèse, Université Bordeaux 1 (juillet 2000).

[Ha] U. Hamenstädt, New examples of maximal surfaces. Enseign. Math. 47 (2001), 65-101. MR 1844895

[Je] F. W. Jenni, Über den ersten Eigenwert des Laplace-Operators auf ausgewählten Beispielen kompakter Riemannscher Flächen. Comment. Math. Helv. 59 (1984), 193-203. Zbl 0541.30034 MR 0749104

[Ku] I. Kuribayashi, A. Kuribayashi, Automorphism groups of compact Riemann surfaces of genera three or four. J. Pure Appl. Algebra 65 (1990), 277-292. Zbl 0709.30036 MR 1072285

[Mu] D. Mumford, A remark on Mahler's compactness theorem. Proc. Amer. Math. Soc. 28 (1971), 289-294. Zbl 0215.23202 MR 0276410

[R-R] G. Riera, R. E. Rodrìguez, The period matrix of Bring's curve. Pacific J. Math. 154 (1992), 179-200. Zbl 0734.30036 MR 1154738

[Sc1] P. Schmutz Schaller, Riemann surfaces with shortest geodesic of maximal length. Geom. Funct. Anal. 3 (1993), 564-631. Zbl 0810.53034 MR 1250756

[Sc2] P. Schmutz Schaller, Systoles on Riemann surfaces. Manuscripta Math. 85 (1994), 429-447. Zbl 0819.30027 MR 1305753

[Sc3] P. Schmutz Schaller, Congruence subgroups and maximal Riemann surfaces. J. Geom. Anal. 4 (1994), 207-218. Zbl 0804.3201 MR 1277506

[Sc4] P. Schmutz Schaller, Perfect non-extremal Riemann surfaces. Canad. Math. Bull. 43 (2000), 115-125. Zbl 1041.11027 MR 1749958

[Wo] S. Wolpert, On the symplectic geometry of deformations of a hyperbolic surface. Ann. of Math. 117 (1983), 207-234. Zbl 0518.30040 MR 0690844

Received March 29, 2000; revised October 8, 2002

Alexandre Casamayou-Boucau, 48 Rue Sainte Geneviève, 33800 Bordeaux, France

E-mail: alexandre.casamayou@m4x.org 